\title{
El impacto de las variaciones en la morfología floral sobre las visitas de polinizadores y el éxito reproductivo del arbusto Cytisus scoparius
}

\author{
Melina E. Zuliani ${ }^{1}$ \& Alejandro G. Farji-Brener ${ }^{2,}$ \\ ${ }^{1}$ Departamento de Análisis de Sistemas Complejos. Fundación Bariloche-CONICET. ${ }^{2}$ Laboratorio de Investigaciones en \\ Hormigas (LIHO)- INIBIOMA-CONICET y CRUB-Universidad Nacional del Comahue.
}

\begin{abstract}
Resumen. Conocer el efecto de la morfología floral sobre las visitas de los polinizadores y sus consecuencias en la reproducción es vital para comprender la ecología y la evolución de las interacciones planta-polinizador. Determinamos si las variaciones en el área y la simetría del estandarte floral afectan la frecuencia de visitas y la producción de semillas en el arbusto Cytisus scoparius (L.) Link (retama). Seguimos en el tiempo una numerosa cantidad de flores que presentaban variación natural en el área y la simetría de sus estandartes, y realizamos experimentos manipulativos a campo en los que modificamos dichas características. Finalmente, determinamos la frecuencia de visitas y la producción de semillas por fruto. Mientras el estandarte mostró una distribución de frecuencias con una gran mayoría de casos de simetría casi perfecta, su área se distribuyó en una curva normal más aplanada, con gran frecuencia de casos alrededor del promedio. Las flores visitadas durante el muestreo presentaron estandartes más simétricos, pero de área similar que las no visitadas. De acuerdo con esto, la probabilidad de visita se asoció con la simetría del estandarte, pero no con su área. Por último, el experimento a campo mostró que las flores con estandarte reducido al 50\%, pero que mantenía su simetría, fueron mucho más visitadas y produjeron más semillas/fruto que aquellas con el estandarte reducido al 50\%, pero que no mantenían la simetría. Estos resultados sugieren que, en la retama, la simetría del estandarte es un aspecto clave de la morfología floral para atraer polinizadores. La reducida tasa de visita a flores con estandartes muy asimétricos se podría deber a una reducción en el reconocimiento floral, o a que los visitantes lo perciben como un signo de daño y lo interpretan como una señal de bajo nivel de recompensas. Este trabajo resalta la importancia de la simetría como señal atractiva para los polinizadores e ilustra cómo la variación de estos rasgos dentro de una misma especie puede afectar el éxito reproductivo y, por ende, estar sujetos a selección natural.
\end{abstract}

[Palabras clave: estandarte floral, Patagonia, polinización, retama, simetría floral]

Aвstract. The impact of variations in floral morphology on pollinator visits and reproductive success of the Cytisus scoparius shrub. To know the effect of floral morphology on the visitation frequency of pollinators and their consequences on reproduction is key to understand the ecology and evolution of plant-pollinator interactions. We determined if variations in the area and symmetry of the floral banner affect the frequency of pollinator visits and seed production in the shrub Cytisus scoparius (L.) Link (broom). During the flowering period, we followed a large number of flowers that presented natural variation in the area and symmetry of their banners, and we carried out manipulative experiments in the field modifying these characteristics. Finally, we determined the visitation frequency and the seed production/fruit of each flower. While the symmetry of the banner showed a frequency distribution with a vast majority of cases centered in almost perfect symmetry, the banner area presented a flatter normal curve, with a high frequency of cases around the mean. The visited flowers along the sampled period showed more symmetrical banners, but with similar area than the unvisited flowers; the probability of visits were associated with the symmetry of the banner, but not with its area. The field experiment showed that flowers with a banner reduced to $50 \%$, but maintaining their symmetry, were much more visited and produced more seeds/fruit than those with the banner reduced to $50 \%$ without maintaining its symmetry. These results suggest that, in Cytisus scoparius, the symmetry of the banner is a key aspect of floral morphology to attract pollinators. The low rate of visited flowers with highly asymmetric banners could be due to a reduction in floral recognition or because it is perceived by visitors as a flower damage, which may be interpreted as a sign of a low reward level. This work highlights the importance of symmetry as an attractive signal for pollinators, and illustrates how the variation of these traits within the same species can affect reproductive success, and therefore be subject to natural selection.

[Keywords: floral banner, floral symmetry, Patagonia, pollination]

Editor asociado: Diego Vázquez

$\bar{\triangle}$ alefarji@yahoo.com
Recibido: 12 de Mayo de 2020

Aceptado: 13 de Julio de 2020 


\section{INTRODUCCIÓN}

Las plantas con flor que dependen de polinizadores para su reproducción enfrentan un problema: ofrecen recompensas - como néctar y polen - que los visitantes florales no pueden detectar directamente. Por ende, las flores evolucionaron con ciertos rasgos que funcionan como señales de la existencia de sus recompensas. Estos rasgos florales son los que los polinizadores pueden percibir directamente; normalmente 'comunican' el status de la recompensa o facilitan el contacto de los visitantes con las estructuras reproductivas de la flor (Knauer and Schiestl 2014). Entonces, conocer la variación de estas estructuras florales y su efecto atractivo sobre los visitantes es vital para comprender la ecología y la evolución de las interacciones planta-polinizador.

La variación intraespecífica de las características de la flor es un buen escenario para estudiar la relación entre las señales florales y las visitas de polinizadores (Herrera et al. 2006). Por un lado, las variaciones intraespecíficas son sencillas de detectar, pueden ser percibidas por los polinizadores, $\mathrm{y}$ su importancia está bien documentada (Galen 1989; Nuttman et al. 2006; Papiorek et al. 2013; Shrestha et al. 2016). Por otra parte, como estas variaciones ocurren dentro de la misma especie, no es necesario emplear controles filogenéticos. Adicionalmente, diferentes morfos florales pueden crecer muy cercanos entre sí, compartiendo las mismas condiciones ambientales y bióticas, lo cual facilita su comparación. Finalmente, los polinizadores pueden percibir cambios intraespecíficos en los rasgos florales y responder modificando su tasa de visitas (Nuttman et al. 2006). Por todas estas características, el estudio de las variaciones de los rasgos florales dentro de una misma especie es un campo prometedor y valioso para comprender las interacciones planta-polinizador (Herrera et al. 2006).

Dentro de una misma especie, numerosas características de las flores como el color, el tamaño y la simetría pueden indicar el estado de la recompensa o facilitar el acceso de los visitantes a la flor (Orbán and Plowright 2014). Por ejemplo, en Alkanna orientalis (Boraginaceae) los polinizadores prefieren visitar aquellas flores con colores más brillantes por sobre las más opacas porque las primeras poseen mayor cantidad de néctar que las segundas (Nuttman et al. 2006). Flores con guías de néctar más claramente marcados son, en general, preferidas (Waser and Price 1985). En Arnebia szechenyi (Boraginaceae), las guías de néctar van perdiendo su color cuando las flores ya no tienen recompensa, por lo cual reciben escasas visitas (Zhang et al. 2017). También es común que, dentro de una misma especie, los polinizadores elijan visitar aquellas flores con corolas más grandes que, por lo general, poseen más néctar y polen que las flores de corolas más pequeñas (Stanton and Preston 1988: Campbell et al. 1991; Cohen and Shmida 1993; Blarer et al. 2002). La simetría floral también es un rasgo relevante que incrementa la tasa de visitas de los polinizadores; normalmente se la asocia con un buen estado de salud de la planta y con un mejor status de la recompensa (Møller 1995; Neal et al. 1998; Guirfa et al. 1999). En resumen, dentro de una misma especie es común que las flores con corolas más grandes, más simétricas y de colores más vivos o con marcas que faciliten el acceso a la flor sean visitadas especialmente.

Cytisus scoparius (L.) (i.e., retama) es un arbusto que representa un modelo de estudio ideal para analizar cómo las variaciones en la morfología floral pueden afectar la visita de los polinizadores. Primero, este arbusto es muy abundante, sencillo de localizar, con flores de color intenso fáciles de observar, y muy visitadas por los polinizadores. Segundo, parte de su morfología floral (en particular, su pétalo superior, denominado estandarte) tendría una potencial función atractiva para los visitantes florales (Parker 1997; Bode and Tong 2018); las variaciones en la morfología del estandarte podrían influir, entonces, en la tasa de visita de los polinizadores (Susuki 2003). Tercero, el estandarte de C. scoparius presenta variación natural en su área y simetría, y por su tamaño se lo puede manipular fácilmente sin dañar la flor. Además, las flores con variaciones en la simetría y el área de su estandarte se encuentran normalmente entremezcladas dentro de la misma planta o parche floral, lo que permite realizar comparaciones a campo. Cuarto, sus polinizadores más frecuentes son las abejas (Apis melífera) y abejorros (Bombus spp.), que suelen ser sensibles a las variaciones morfológicas de las flores que visitan (Neal et al. 1998; Guirfa et al. 1999; Orbán and Plowright 2014). Quinto, las flores sólo pueden ser abiertas si son estimuladas por los visitantes florales; por esto, la apertura de las flores es una señal inequívoca de que fue visitada por un polinizador (Parker 1997; Morales and Aizen 2002; Gavini and 
Farji-Brener 2015; Vidal and Farji-Brener 2020). Por último, estimar su reproducción es relativamente simple. Sus frutos son una cápsula en forma de legumbre que permite contabilizar de forma sencilla la cantidad de semillas antes de su dehiscencia explosiva (Parker 1997). En resumen, la retama es un excelente candidato para estudiar cómo los cambios en la morfología floral afectan las visitas y el éxito reproductivo debido a que es muy abundante, a las características de sus flores, a la facilidad de su manipulación a campo y a la factibilidad para estimar su adecuación.

El objetivo de este trabajo fue determinar si variaciones en el área y la simetría del estandarte en las flores de Cytisus scoparius afectaban la frecuencia de las visitas y la adecuación del arbusto. Para ello, seguimos en el tiempo una numerosa cantidad de flores que presentaban variación natural en el área y simetría de sus estandartes, y realizamos experimentos manipulativos a campo modificando dichas características. En ambos casos, determinamos la frecuencia de visitas y la producción de semillas por fruto. Dependiendo si el área o la simetría del estandarte eran relativamente más relevantes como señal para los polinizadores, tanto en el enfoque comparativo como en el experimental esperábamos que las flores más visitadas y las que posean mayor cantidad de semillas/fruto sean aquellas con áreas mayores o con mayores niveles de simetría, respectivamente. Estas predicciones se encuentran desarrolladas con mayor detalle en la sección de metodología.

\section{Materiales y Métodos}

\section{Sitio y especie de estudio}

Realizamos el muestreo desde mediados de la primavera hasta el fin del verano de 2020 (de noviembre a fin de febrero) en diferentes sitios de matorral con gran abundancia de plantas de Cytisus scoparius, ubicados en las afueras de la ciudad de San Carlos de Bariloche, Argentina $\left(41^{\circ} 09^{\prime} \mathrm{S}-71^{\circ} 18^{\prime} \mathrm{O}\right)$. La precipitación anual del área es de $\sim 1600 \mathrm{~mm}$ y la temperatura media es $2{ }^{\circ} \mathrm{C}$ en invierno y $13{ }^{\circ} \mathrm{C}$ en verano. Cytisus scoparius es un una especie arbustiva originaria de Europa; pertenece a la familia Fabaceae, y es muy abundante en el noroeste de la Patagonia. Trabajamos con los arbustos que presentan el morfo floral amarillo, dado que es el más abundante y más visitado por los polinizadores en el área de estudio
(Gavini and Farji-Brener 2015). Sus flores papilionadas están compuestas por 5 pétalos. El pétalo superior se denomina estandarte, los dos laterales se llaman alas y los dos pétalos inferiores forman una quilla (Figura 1A). El estandarte presenta simetría bilateral, y tal como su nombre lo sugiere, funcionaría como atrayente para los visitantes florales (Gavini and Farji-Brener 2015; Bode and Tong 2018). El mecanismo de presentación del polen es explosivo. Los estambres y el pistilo están sometidos a una gran tensión bajo la quilla y son expulsados cuando el polinizador ejerce presión. Al visitar las flores, el polinizador estimula la apertura explosiva de los pétalosquilla mediante zumbidos y movimientos, liberando a los estambres y el pistilo, por lo que la parte ventral del animal queda impregnada de polen. En consecuencia, si la flor está cerrada significa que aún no fue visitada, y si está abierta, con los estambres y el pistilo expuestos, implica que la flor ya fue visitada (Parker 1997; Suzuki 2003). Su sistema reproductivo es auto-incompatible. La polinización cruzada incrementa varias veces su producción de semillas, por lo cual se deduce que esta especie se encuentra limitada por polen (Parker 1997; Rodríguez-Riaño et al. 1999). Los agentes polinizadores más comunes son himenópteros; los más frecuentes son las
A)

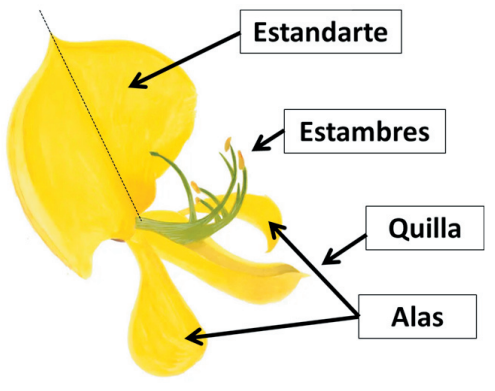

B)
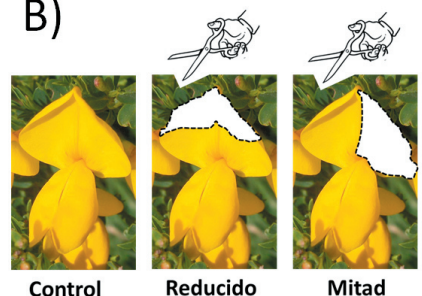

Mitad

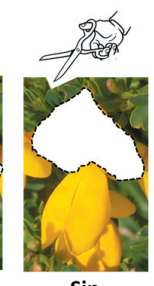

Sin
Figura 1. A) Dibujo de la flor del arbusto Cytisus scoparius (retama) con la denominación de sus pétalos. Se detalla la simetría bilateral del estandarte. B) Descripción de los tratamientos del experimento a campo, en donde se modifica el área y la simetría del estandarte.

Figure 1. Drawing of the flower of the shrub Cytisus scoparius (broom) with the denomination of its petals. Bilateral symmetry of the banner is detailed. B) Description of the treatments of the field experiment, where the area and symmetry of the banner are modified. 
abejas (Apiadeae) y los abejorros (Bombideae). El fruto es una cápsula en forma de legumbre de dehiscencia explosiva.

\section{Metodología}

\section{Seguimiento de flores en su estado natural}

Para determinar el efecto de las variaciones en el área y la simetría del estandarte sobre las visitas de los polinizadores y la producción de semillas, realizamos dos tipos de mediciones: seguimos en el tiempo 200 flores que presentaban variación natural en el área y simetría de sus estandartes, y realizamos un experimento a campo en el que manipulamos el área y la simetría del estandarte en 400 flores. En ambos casos determinamos el efecto de estas variaciones (naturales y manipuladas) sobre las visitas de los polinizadores y la producción de semillas por fruto. Para la primera parte seleccionamos al azar 200 flores cerradas (i.e., no visitadas). Estas flores estaban ubicadas entre los 1 y $2 \mathrm{~m}$ de altura en diferentes parches florales, con vecindarios similares en cuanto a densidad de flores cercanas. A cada flor le medimos su área y simetría del estandarte, la marcamos individualmente y la visitamos cada 5 días durante todo el período de floración hasta el período de fructificación. Al final de la floración, cada flor fue caracterizada como visitada o no visitada (i.e., abierta o cerrada, respectivamente). El área y la simetría de los estandartes fueron calculados a través de fotografías digitales mediante el programa Image ${ }^{\circledR}$. Las fotos fueron sacadas de frente para evitar distorsiones y con una escala para poder realizar las estimaciones en $\mathrm{cm}^{2}$. La simetría del estandarte fue estimada superponiendo digitalmente las mitades laterales del mismo y estimando el área sobresaliente, la cual fue considerada como un índice de asimetría. Dada la clara simetría bilateral del estandarte (Figura 1A), este indicador de asimetría tomaba valores de cero para simetría perfecta (i.e., área sobresaliente nula al superponer ambas mitades del estandarte), aumentando en valor a mayores niveles de asimetría. El área y la simetría del estandarte entre flores visitadas y no visitadas fue comparada mediante pruebas $t$. Adicionalmente, para determinar si variaciones en el área o en la simetría afectaban la probabilidad de visita se realizaron regresiones logísticas, en donde la variable respuesta binaria fue flor visitada (i.e., abierta $=1$ ) o no visitada (i.e., cerrada $=0$ ) y la variables independientes continuas fueron el área del estandarte y su simetría.

\section{Experimento manipulativo a campo}

Para determinar de manera experimental el efecto del área y la simetría del estandarte sobre las visitas y su efecto sobre la adecuación de la retama, se realizó el siguiente experimento. Se seleccionaron un total de 400 flores al azar de diferentes parches florales ubicados en 4 sitios separados entre sí en $\sim 150 \mathrm{~m}$ (100 flores por sitio). En cada sitio se signaron al azar cuatro tratamientos a las 100 flores (25 flores por tratamiento). Dentro de cada sitio, las 100 flores con los diferentes tratamientos se encontraban entremezcladas entre sí, inmersas en un vecindario floral con similar densidad de flores. Los tratamientos fueron: control (flores sin manipulación alguna del estandarte), reducido (flores a las cuales se les recortó con cuidado la parte superior del estandarte para reducir en $50 \%$ su área, pero manteniendo su forma y simetría), mitad (flores a las cuales se les cortó una de las mitades laterales del estandarte - alternativamente derecho e izquierdo-, reduciendo al 50\% su área total, pero sin mantener su simetría) y sin, en el que se eliminó por completo el estandarte (Figura 1B). De esta manera pretendíamos separar el efecto del área del de la simetría. Si lo atractivo para los polinizadores era sólo el área total del estandarte, nosotros esperamos que la visita de los polinizadores en los tratamientos reducido y mitad fuese semejante, dado que en ambos casos el estandarte poseía la misma área. En particular, esperábamos que las visitas disminuyan en el siguiente orden: control> reducido $=$ mitad $>\sin$. Por otra parte, si sólo la simetría del estandarte era una señal relevante para los polinizadores (y no necesariamente el área), esperábamos que los tratamientos control y reducido tuviesen similar número de visitas (ya que ambos poseen simetría perfecta), mayor que el tratamiento mitad. En particular, esperábamos que las visitas disminuyan en el siguiente orden:control $=$ reducido $>$ mitad $>$ sin. Si ambos factores eran relevantes, dado que las flores control presentaban más área de estandarte simétrico, esperábamos que la visita decreciera en el siguiente orden: control $>$ reducido $>\operatorname{mitad}>\sin$. Finalmente, si el estandarte no cumple ningún papel en la atracción de los polinizadores, esperábamos que todos los tratamientos presentaran la misma frecuencia de visitas entre sí. Las 400 flores fueron monitoreadas durante todo el período de floración hasta su senescencia o fructificación, las que finalmente se caracterizaron como visitadas (i.e., abiertas) o no visitadas (i.e., cerradas). En el caso de las flores polinizadas, al final de la temporada de 
verano y antes de que los frutos dispersaran sus semillas de manera explosiva recolectamos las vainas y contabilizamos el número de semillas/fruto. De esta forma pudimos asignar a cada tratamiento la frecuencia de visita así como el promedio de semillas producidas. Para determinar si la frecuencia de visita era independiente de los tratamientos se realizó una tabla de contingencia que fue analizada mediante una prueba $X^{2}$. Los resultados de la producción de semillas/fruto fueron analizado mediante un análisis de varianza de dos vías, en donde se incluyó el sitio ( $n=4$ sitios) como factor aleatorio y los tratamientos (4 niveles) como factor fijo. Las diferencias significativas entre tratamientos fueron analizadas mediante una prueba a posteriori de Tukey. Dado que las flores cerradas no producen frutos, realizamos este análisis dos veces: incluyendo todas las flores (abiertas y cerradas) e incluyendo sólo las flores visitadas (abiertas).

\section{Resultados}

\section{Seguimiento de flores en estado natural}

Las flores marcadas a campo presentaron variación en el área y simetría del estandarte. Por un lado, el área del estandarte varió un orden de magnitud, entre 1.35 a $13.2 \mathrm{~cm}^{2}$, con una distribución normal (KolmogorovSmirnov $d=0.07, P>0.20$ ) alrededor de un promedio de $5.2 \mathrm{~cm}^{2}$. Por otra parte, la asimetría varió entre 0 y $3.5 \mathrm{~cm}^{2}$, mostrando una distribución muy centrada en valores cercanos a cero, con una leve cola hacia la derecha con muy baja frecuencia de casos (Figura 2). De las 200 flores cerradas que fueron observadas durante la temporada de floración, 41 no fueron visitadas y 159 fueron visitadas. La visita de los polinizadores dependió de la simetría del estandarte, pero no de su área (Figura 3). Las flores visitadas presentaron menor asimetría en el estandarte que las no visitadas $(0.30 \pm 0.05$ versus $0.67 \pm 0.14$, media \pm error estándar, $t=3.14, P=0.002$, g.l.=198, respectivamente). Mientras que el área del estandarte entre las flores visitadas y no visitadas fue similar (5.3 \pm 0.2 versus $5.1 \pm 0.4, t=0.41, P=0.68$, g. $1 .=198$, respectivamente). De acuerdo con esto, la probabilidad de que una flor sea visitada no dependió del área del estandarte, pero sí de su nivel de asimetría (regresión logística, $n=200$ flores, $X^{2}=0.2, P=0.68$ y $X^{2}=8.5, P=0.003$, g.l.=1, respectivamente). Específicamente, las flores con mayor nivel de asimetría tuvieron menos probabilidad de ser visitadas (Figura 4).

\section{Experimento manipulativo a campo}

La cantidad de flores abiertas al final del experimento dependió de los tratamientos recibidos. En particular, las flores control y aquellas con el estandarte reducido recibieron muchas más visitas que aquellas con medio estandarte y sin estandarte. Mientras el $82 \%$ las flores control y el $67 \%$ de las flores con el
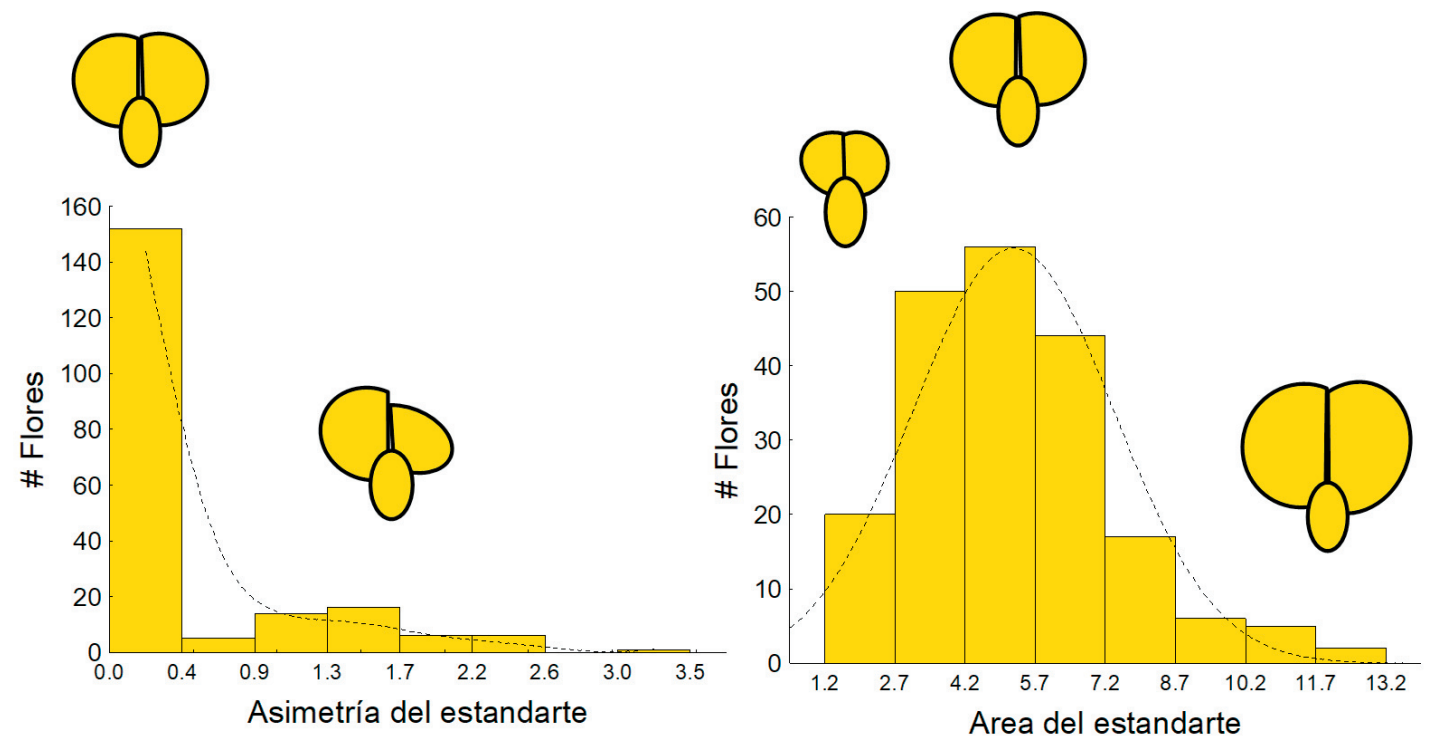

Figura 2. Distribución de frecuencias de la asimetría y el área del estandarte $\left(\mathrm{cm}^{2}\right)$ en 200 flores del arbusto Cytisus scoparius (retama).

Figure 2. Frequency distribution of the asymmetry and banner area $\left(\mathrm{cm}^{2}\right)$ in 200 flowers of the shrub Cytisus scoparius (broom). 


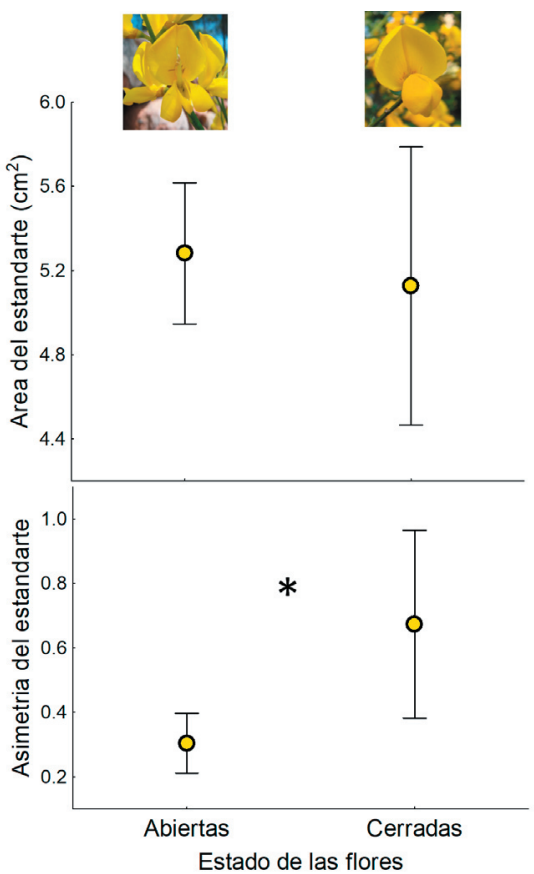

Figura 3. Comparación entre el área y la asimetría del estandarte (promedios e intervalos del $95 \%$ de confianza) entre flores visitadas (i.e., abiertas) y no visitadas (i.e., cerradas) del arbusto Cytisus scoparius (retama). *: Diferencias estadísticamente significativas.

Figure 3. Comparison between the area and asymmetry of the banner (means and 95\% confidence intervals) between visited (i.e., open) and unvisited (i.e., closed) flowers of the Cytisus scoparius (broom) shrub. *:Statistically significant differences.

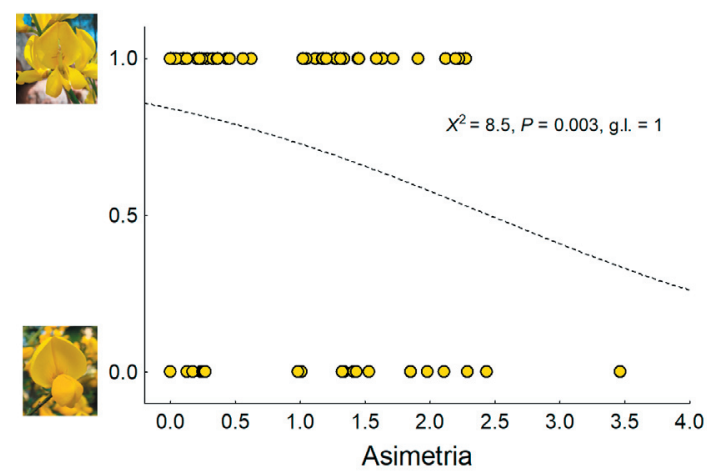

Figura 4. Probabilidad de que una flor sea visitada (1) o no visitada (0) en relación con su nivel de asimetría $\left(\mathrm{cm}^{2}\right)$ en el arbusto Cytisus scoparius (retama).

Figure 4. Probability that a flower was visited (1) or not (0) in relation to its level of asymmetry $\left(\mathrm{cm}^{2}\right)$ in the Cytisus scoparius shrub (broom).

estandarte reducido recibieron visitas, sólo $8 \%$ de las flores con medio o sin estandarte fueron visitadas $\left(X^{2}=184\right.$, g.l. $\left.=3, P<0.0001\right)$. Considerando todas las flores (abiertas y cerradas), el número promedio de semillas/ fruto también varió entre tratamientos $\left(F_{3,392}=101, P<0.001\right)$, pero no entre sitios $\left(F_{3,392}=2.02, P=0.10\right)$. Las flores sin manipular tuvieron un mayor número de semillas/fruto que aquellas con el estandarte reducido (8.6 \pm 0.5 versus $6.1 \pm 0.5$, respectivamente; mediaterror estándar), las cuales a su vez

\section{B)}

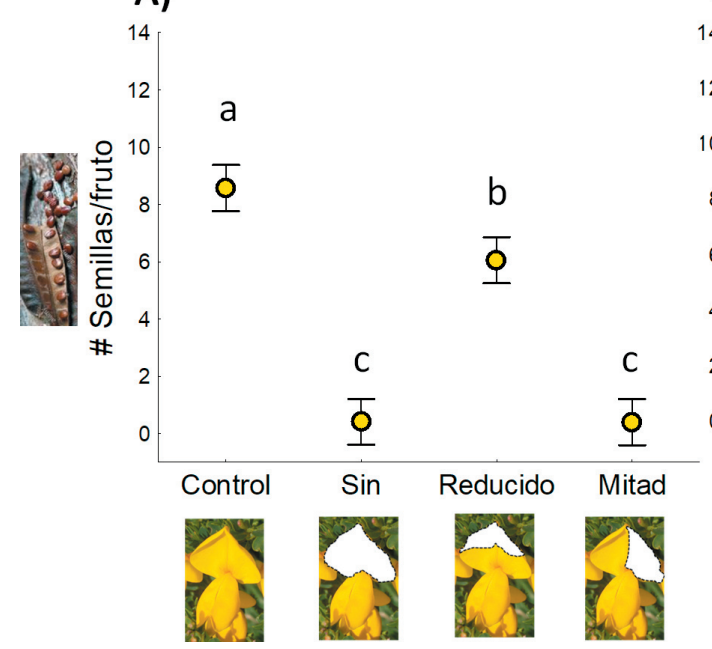

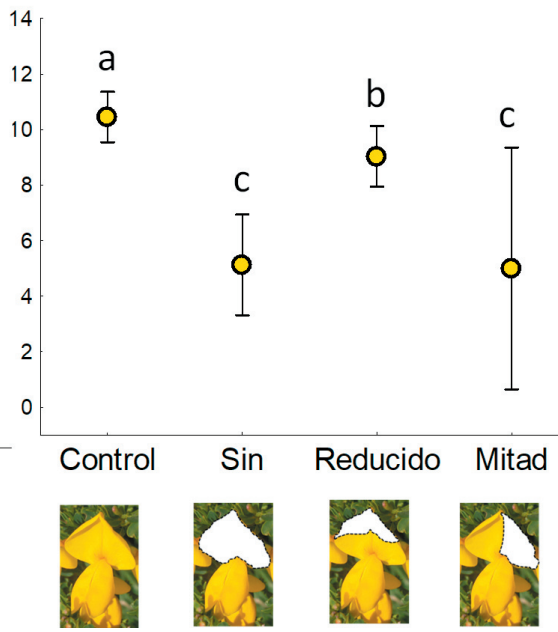

Figura 5. Cantidad de semillas/fruto (media e intervalo de confianza del 95\%) en frutos provenientes de flores cuyos estandartes fueron reducidos a la mitad sin mantener su simetría (mitad) y manteniendo su simetría (reducido), removidos por completo (sin) y sin manipular (control) en el arbusto Cytisus scoparius (retama). A) Considerando las flores visitadas y no visitadas. B) Considerando sólo las flores visitadas. Letras diferentes indican diferencias estadísticamente significativas (prueba a posteriori de Tukey, $P<0.05$ ).

Figure 5. Number of seeds/fruit (mean and 95\% confidence interval) in fruits from flowers whose banners were reduced by half without maintaining their symmetry (half) and maintaining their symmetry (reduced), completely removed (without) and without manipulate (control) in shrubs of Cytisus scoparius (broom). A) Considering visited and not visited flowers. B) Only considering the visited flowers. Different letters indicate statistically significant differences (Tukey's posterior test, $P<0.05$ ) 
tuvieron un mayor número de semillas/fruto que las flores con medio estandarte $(0.4 \pm 0.2)$ y sin estandarte $(0.41 \pm 0.15)$, que fueron similares entre sí (todas comparaciones estadísticamente significativas, prueba a posteriori de Tukey, $P<0.05$ ) (Figura 5A). Esta tendencia se mantuvo al analizar sólo las flores que fueron visitadas, que difirieron entre tratamientos $\left(F_{3157}=4.4\right.$, $P<0.005)$, pero no entre sitios $\left(F_{3,157}=2.1, P=0.09\right)$. Las flores sin manipular tuvieron un mayor número de semillas/fruto que aquellas con el estandarte reducido ( $10.5 \pm 0.5$ versus $9 \pm 0.5$, respectivamente; mediaterror estándar), las que, a su vez, tuvieron un mayor número de semillas/fruto que aquellas flores con medio estandarte $(5 \pm 1.8)$ y sin estandarte $(5.1 \pm 0.8)$, las cuales fueron similares entre sí (todas comparaciones estadísticamente significativas, prueba a posteriori de Tukey, $P<0.05$ (Figura 5B). Estos resultados fueron consistentes entre los diferentes sitios, factor que no fue estadísticamente significativo.

\section{Discusión}

La variación natural del área y simetría de los estandartes, el seguimiento de las visitas a campo y los resultados del experimento sugieren que la simetría, más que el área, es el rasgo más relevante del estandarte que afecta la visita de los polinizadores. Primero, la curva de distribución de la simetría del estandarte presentó un pico muy pronunciado en el valor esperado para simetría perfecta, con muy pocos casos lejos del promedio, mientras que la distribución de frecuencias del área del estandarte mostró una gran cantidad de casos alrededor del promedio. Segundo, la probabilidad de que una flor fuese visitada en condiciones naturales dependió de la simetría del estandarte, pero no de su área. Finalmente, antefloresqueexperimentalmentepresentaban similar área de estandarte, pero diferían en su simetría, los polinizadores visitaron más las flores con estandartes simétricos, las que, además, produjeron más semillas. Todos estos resultados sugieren que la simetría del estandarte juega un papel clave en la atracción de la flor de la retama.

Mientras la distribución de la asimetría del estandarte presentó casi el $80 \%$ de los casos cercano a simetría perfecta y muy baja frecuencia de casos de estandartes asimétricos, la distribución del área del estandarte mostró una curva normal aplanada, con una gran cantidad de casos alrededor del promedio. Por un lado, esto sugiere una alta presión selectiva hacia una simetría perfecta, en la que grandes asimetrías serían penalizadas por la selección natural (Guirfa et al. 1999; Møller 2000). Y por otra parte, el área del estandarte parecería estar sujeto a una selección de tipo estabilizadora, con una alta cantidad de casos alrededor del promedio $\left(\sim 5 \mathrm{~cm}^{2}\right)$, en los que sólo se penalizarían los casos muy extremos de estandartes demasiado pequeños $(<2.5$ $\mathrm{cm}^{2}$ ) o demasiado grandes $\left(>9 \mathrm{~cm}^{2}\right)$. Los estandartes demasiado pequeños podrían no llegar a ser una señal visual suficiente para atraer polinizadores (Bode and Tong 2018), mientras que los demasiado grandes podrían implicar un costo excesivo de construcción, ser más susceptibles al efecto del viento o a encorvarse, ocasionando daños u obstruyendo la estructura floral. En resumen, la distribución de frecuencias de las variaciones naturales del área y la simetría sugieren que la simetría, más que el área per se, sería el rasgo del estandarte más atractivo para los visitantes florales.

Tanto los resultados de las flores que fueron monitoreadas en condiciones naturales como aquellos provenientes del experimento sugieren que la simetría del estandarte es clave en determinar la visita de una flor. Por un lado, las flores visitadas presentaron estandartes con el doble de simetría que las no visitadas, pero sus áreas fueron similares. Según esto, la probabilidad de que una flor sea visitada dependió del nivel de simetría del estandarte, pero no de su área. Por otra parte, ya sea considerando todas las flores o sólo aquellas que fueron visitadas, tanto la frecuencia de visita como la producción de semillas/fruto fue mucho mayor en aquellas flores que mantenían la simetría de los estandartes respecto a aquellas que no. En particular, la diferencia en la producción de semillas/fruto entre flores cuyos estandartes eran simétricos, pero que diferían en área (e.g., control versus reducida) siempre fue menor que la diferencia entre las flores cuyos estandartes eran de similar área, pero diferían en su simetría (e.g., reducida versus mitad) (en promedio, entre 1-2 semillas más por fruto versus 4-5 semillas más por fruto). Nosotros no estamos sugiriendo que el área del estandarte no influye sobre la tasa de visita. En términos relativos, resulta obvio que estandartes muy reducidos o ausentes no atraen polinizadores, y que a nivel de simetría semejante, estandartes mayores logran una leve mayor producción de semillas. Pero todos los resultados son consistentes y sugieren que, en términos comparativos, los 
polinizadores evaden con más frecuencia visitar flores con estandartes asimétricos que flores con estandartes reducidos.

Varios mecanismos no exclusivos podrían explicar el motivo por el cual los visitantes florales evaden visitar flores con estandartes muy asimétricos. Primero, se sabe que para los himenópteros, la simetría juega un papel muy importante en el reconocimiento floral (Neal et al. 1998; Guirfa et al. 1999; Gong and Huang 2009). Flores con gran asimetría pueden no ser reconocidas y confundidas con flores de especies poco preferidas. Además, estos cambios en la morfología floral podrían atraer a polinizadores no especialistas que pueden ser incapaces de abrir el mecanismo explosivo de la quilla. Segundo, como ya fue discutido, la simetría puede ser una señal honesta del estado de la recompensa (Møller 1995). En este caso, los polinizadores podrían interpretar grandes asimetrías en el estandarte como una señal de baja disponibilidad de recompensas y preferir visitar otra flor. Los polinizadores podrían interpretar asimetrías en el estandarte como un daño en los pétalos de la flor ocasionado por herbívoros (i.e., florivoría) (McCall and Irwin 2006). Se conoce que los daños en los pétalos pueden generan efectos negativos sobre la calidad y la cantidad de recompensas que ofrece una planta (Krupnick et al. 1999; McCall 2008; Cascante-Marín et al. 2009), incluyendo la calidad del polen (Aizen and Raffaele 1998), que es la recompensa típica de la retama. Por ende, los polinizadores evitarían visitar una flor que interpretan como dañada $\mathrm{y}$, por lo tanto, con bajo nivel de recompensas. Todos estos mecanismos pueden actuar en conjunto para determinar los motivos por los cuales las flores asimétricas son poco visitadas por los polinizadores, y producen poca cantidad de semillas por fruto.

Este trabajo presenta ciertas debilidades, pero también fortalezas. Primero, no se discriminan los mecanismos descriptos en el párrafo anterior. Futuros experimentos deberían realizarse para determinar si grandes niveles de asimetría ocasionan una reducción en el reconocimiento floral, una atracción para polinizadores de baja calidad, o es interpretado por los visitantes como una señal de un bajo nivel de recompensas. Segundo, las variaciones en la simetría del estandarte realizadas de manera experimental (esto es, eliminar por completo una mitad) se podría consider como excesiva. Sin embargo, la eliminación de la mitad de un estandarte de área promedio $\left(\sim 5 \mathrm{~cm}^{2}\right)$ genera una asimetría de $2.5 \mathrm{~cm}^{2}$, la cual - aunque con baja frecuencia - existe en condiciones naturales. Sería interesante generar experimentalmente asimetrías menos extremas en el estandarte para verificar el patrón aquí descubierto. Finalmente, la adecuación es estimada sólo por la producción de semillas y no por la exportación de polen. Es sabido que para medir por completo el éxito reproductivo de una planta es recomendable estimar tanto la adecuación femenina como la masculina (Sutherland and Delph 1984). Sin embargo, y aunque no fue medido de forma directa en este trabajo, es factible que los resultados de la exportación de polen sean similares a los encontrados en la producción de semillas/fruto. Dado que en la retama el polen se libera de forma explosiva, la primera visita es la que transporta casi todo el polen (Parker 1997; Susuki 2003). En consecuencia, y para esta especie en particular, la frecuencia de visita (i.e., la frecuencia de flores abiertas) podría considerarse como un estimador de la exportación de polen, y por ende, de la adecuación masculina de la planta (Parker 1997). Más allá de estas debilidades, consideramos que el trabajo presenta varias fortalezas. Por un lado, los resultados de los enfoques comparativos y experimentales son consistentes. Tanto la distribución de las variaciones morfológicas de los estandartes, como las mediciones de las visitas a flores en su estado natural, como los resultados del experimento sugieren que la simetría es un rasgo clave del estandarte en atraer visitantes florales. En adición, nuestro diseño experimental permitió discriminar mediante manipulaciones controladas el efecto del área del estandarte del efecto de su simetría, evitando confusiones en la interpretación de la importancia relativa de ambos factores. Por otra parte, todos estos resultados provinieron de un alto número de flores que representaron una gran variación natural, las cuales fueron seguidas durante todo el período de floración. Por último, este trabajo pudo relacionar la frecuencia de visita con su consecuencia más directa, la producción de semillas. Consideramos que todas estas características hacen válida la interpretación de que la simetría del estandarte cumple un papel clave en la atracción de los polinizadores y la adecuación de la retama. En este sentido, nuestro trabajo aporta nueva información sobre el efecto de la morfología floral sobre la polinización, resaltando la importancia de la simetría como señal atractiva para 
los visitantes florales, e ilustrando cómo la variación de estos rasgos dentro de una misma especie puede afectar el éxito reproductivo y, por ende, estar sujetos a selección natural.

Agradecimientos. Este trabajo es la continuidad de uno de los proyectos finales de la materia "Ecología de las Interacciones Planta-Animal" que se dicta en el Centro
Regional Universitario Bariloche de la Universidad Nacional del Comahue para la Licenciatura y Doctorado en Biología. Versiones preliminares del proyecto fueron beneficiadas por discusiones entre compañeros de la cursada del año 2019. Los comentarios de dos revisores anónimos nos ayudaron a mejorar la versión preliminar de este artículo.

\section{REFERENCIAS}

Aizen, M. A., and E. Raffaele. 1998. Flowering-shoot defoliation affects pollen grain size and postpollination pollen performance in Alstroemeria aurea. Ecology 79:2133-2142. https://doi.org/10.2307/176716. https://doi.org/10.1890/00129658(1998)079[2133:FSDAPG]2.0.CO;2.

Blarer, A., T. Keasar, and A. Shmida. 2002. Possible mechanisms for the formation of flower size preferences by foraging bumblebees. Ethology 108:341-351. https://doi.org/10.1046/j.1439-0310.2002.00778.x.

Bode, R. F., and R. Tong. 2018. Pollinators exert positive selection on flower size on urban, but not on rural Scotch broom (Cytisus scoparius L. Link). J of Plant Ecol 11:493-501. https://doi.org/10.1093/jpe/rtx024.

Campbell, D. R., N. M. Waser, M. V. Price, E. A. Lynch, and R. J. Mitchell. 1991. Components of phenotypic selection: pollen export and flower corolla width in Ipomopsis aggregata. Evolution 45:1458-1467. https://doi.org/10.2307/2409892. https://doi.org/10.1111/j.1558-5646.1991.tb02648.x.

Cascante-Marín, A., J. H. Wolf, and J. G. B. Oostermeijer. 2009. Wasp florivory decreases reproductive success in an epiphytic bromeliad. Plant Ecol 203:149-153. https://doi.org/10.1007/s11258-008-9522-y.

Cohen, D., and A. Shmida. 1993. The evolution of flower display and reward. Evol Biol 27:197-243. https://doi.org/ 10.1007/978-1-4615-2878-4_6.

Galen, C. 1989. Measuring pollinator-mediated selection on morphometric floral traits: bumblebees and the alpine skypilot, Polemonium viscosum. Evolution 43:882-890. https://doi.org/10.1111/j.1558-5646.1989.tb05185.x. https: //doi.org/10.2307/2409315.

Gavini, S. S., and A. G. Farji-Brener. 2015. La importancia del color: morfos florales, tasas de visita y éxito reproductivo en el arbusto Sarothamnus scoparius. Ecología Austral 25:204-211. https://doi.org/10.25260/EA.15.25.3.0.82.

Giurfa, M., A. Dafni, and P. R. Neal. 1999. Floral symmetry and its role in plant-pollinator systems. Int J of Plant Sciences 160:S41-S50. https://doi.org/10.1086/314214.

Gong, Y. B., and S. Q. Huang. 2009. Floral symmetry: pollinator-mediated stabilizing selection on flower size in bilateral species. Proceedings of the Royal Society B: Biological Sciences 276:4013-4020. https://doi.org/10.1098/ rspb.2009.1254.

Herrera, C. M., M. C. Castellanos, and M. Medrano. 2006. Geographical context of floral evolution: towards an improved research programme in floral diversification. Pp. 278-294 in L. Hilton (ed.). Ecology and evolution of flowers. Pp. 377. Oxford University Press, U.K.

Knauer, A. C., and F. P. Schiestl. 2014. Bees use honest floral signals as indicators of reward when visiting flowers. Ecol Letters 18:135-43. https://doi.org/10.1111/ele.12386

Krupnick, G. A., A. E. Weis, and D. R. Campbell. 1999. The consequences of floral herbivory for pollinator service to Isomeris arborea. Ecology 80:125-134. https://doi.org/10.2307/176984. https://doi.org/10.1890/0012-9658(1999)080[0125: TCOFHF]2.0.CO;2.

McCall A. C., and R. E. Irwin. 2006. Florivory: the intersection of pollination and herbivory. Ecol Letters 9:1351-1365. https://doi.org/10.1111/j.1461-0248.2006.00975.x.

McCall, A. C. 2008. Florivory affects pollinator visitation and female fitness in Nemophila menziesii. Oecologia 155:729737. https://doi.org/10.1007/s00442-007-0934-5.

Møller, A. P. 1995. Bumblebee preference for symmetrical flowers. Proc Natl Acad Sci 92:2288-2292. https://doi.org/ 10.1073/pnas.92.6.2288.

Møller, A. P. 2000. Developmental stability and pollination. Oecologia 123:149-157. https://doi.org/10.1007/ s004420051000.

Morales, C. L., and M. A. Aizen. 2002. Does invasion of exotic plants promote invasion of exotic flower visitors? A case study from the temperate forests of the southern Andes. Biol Inv 4:87-100. https://doi.org/10.1023/A: 1020513012689.

Neal, P. R., A. Dafni, and M. Giurfa. 1998. Floral symmetry and its role in plant-pollinator systems: terminology, distribution, and hypotheses. Ann Rev Ecol Syst 29:345-373. https://doi.org/10.1146/annurev.ecolsys.29.1.345.

Nuttman, C. V., F. M. Semida, S. Zalat, and P. G. Willmer. 2006. Visual cues and foraging choices: bee visits to floral color phases in Alkanna orientalis (Boraginaceae). Biol J Linnean Soc 87:427-435. https://doi.org/10.1111/j.10958312.2006.00582.x.

Orbán, L. L., and C. M. Plowright. 2014. Getting to the start line: how bumblebees and honeybees are visually guided towards their first floral contact. Insec Sociaux 61:325-336. https://doi.org/10.1007/s00040-014-0366-2.

Papiorek, S., K. Rohde, and K. Lunau. 2013. Bees' subtle color preferences: how bees respond to small changes in 
pigment concentration. Naturwissenschaften 100:633-643. https://doi.org/10.1007/s00114-013-1060-3.

Parker, I. M. 1997. Pollinator limitation of Cytisus scoparius (Scotch broom), an invasive exotic shrub. Ecology 78:14571470. https://doi.org/10.1890/0012-9658(1997)078[1457:PLOCSS]2.0.CO;2. https://doi.org/10.2307/2266140.

Rodríguez-Riaño, T., A. Ortega-Olivencia, and J. Devesa. 1999. Reproductive biology in two Genisteae (Papilionoideae) endemic of the western Mediterranean region: Cytisus striatus and Retama sphaerocarpa Can J Bot 77:809-820. https: //doi.org/10.1139/cjb-77-6-809.

Shrestha, M., K. Lunau, A. Dorin, B. Schulze, M. Bischoff, M. Burd, and A. G. Dyer. 2016. Floral colors in a world without birds and bees: the plants of Macquarie Island. Plant Biol 18:842-850. https://doi.org/10.1111/plb.12456.

Stanton, M. L., and R. E. Preston. 1988. Ecological consequences and phenotypic correlates of petal size variation in wild radish, Raphanus sativus (Brassicaceae). Am J Bot 75:528-539. https://doi.org/10.1002/j.1537-2197.1988.tb13471.x.

Sutherland, S., and L. F. Delph. 1984. On the importance of male fitness in plants: patterns of fruit-set. Ecology 65 : 1093-1104. https://doi.org/10.2307/1938317.

Suzuki, N. 2003. Significance of flower exploding pollination on the reproduction of the Scotch broom, Cytisus scoparius (Leguminosae). Ecol Res 18:523-532. https://doi.org/10.1046/j.1440-1703.2003.00575.x.

Vidal, V. B., and A. G. Farji-Brener. 2020. Lo esencial es invisible a los ojos (humanos): patrones UV explican la mayor tasa de visita de los polinizadores a las flores amarillas del arbusto Cytisus scoparius. Ecología Austral 30:012-018. https://doi.org/10.25260/EA.20.30.1.0.911.

Waser, N. M., and M. V. Price. 1985. The effect of nectar guides on pollinator preference: experimental studies with a montane herb. Oecologia 67:121-126. https://doi.org/10.1007/BF00378462.

Zhang, C., N. J. Vereecken, L. Wang, B. Tian, A. Dafni, Y. Yang, and Y. Duan. 2017. Are nectar guide colour changes a reliable signal to pollinators that enhances reproductive success? Plant Ecol and Div 10:89-96. https://doi.org/10.1080/ 17550874.2017.1350763. 